\title{
Tapentadol in Cancer Patients with Neuropathic Pain: A Comparison of Methadone, Oxycodone, Fentanyl, and Hydromorphone
}

\author{
Miho Takemura, ${ }^{a}$ Kazuyuki Niki, ${ }^{*, a, b}$ Yoshiaki Okamoto, ${ }^{b}$ Yoshinobu Matsuda,${ }^{c}$ Takahito Omae, ${ }^{c}$ \\ Tatsuya Takagi, ${ }^{d}$ and Mikiko Ueda ${ }^{a}$ \\ ${ }^{a}$ Department of Clinical Pharmacy Research and Education, Osaka University Graduate School of Pharmaceutical \\ Sciences; 1-6 Yamadaoka, Suita, Osaka 565-0871, Japan: ${ }^{b}$ Department of Pharmacy, Ashiya Municipal Hospital; \\ 39-1 Asahigaoka, Ashiya, Hyogo 659-8502, Japan: ${ }^{c}$ Department of Palliative Care, Ashiya Municipal Hospital; 39-1 \\ Asahigaoka, Ashiya, Hyogo 659-8502, Japan: and ${ }^{d}$ Department of Pharmainformatics and Pharmacometrics, Osaka \\ University Graduate School of Pharmaceutical Sciences; 1-6 Yamadaoka, Suita, Osaka 565-0871, Japan. \\ Received March 7, 2021; accepted June 18, 2021
}

Tapentadol has $\mu$-opioid receptor stimulating and noradrenaline reuptake inhibiting properties, and should be effective for neuropathic pain (NP). However, the efficacy of tapentadol for NP in cancer patients is unclear. Ashiya Municipal Hospital (Hyogo, Japan) enrolled five groups of Japanese cancer patients between January 1, 2013, and December 31, 2019. Patients with NP were administered tapentadol $(n=29)$, methadone $(n=32)$, oxycodone $(n=20)$, fentanyl $(n=26)$, or hydromorphone $(n=20)$. The primary endpoint was the difference in the verbal rating scale (VRS) scores between days 0 and 7. The secondary endpoint was the tolerability of each opioid. Before administering opioids among the five groups, there was no significant difference in the VRS score $(p=0.99)$. The mean reduction in the VRS score on day 7 was significantly greater in the tapentadol group than in the oxycodone group $(p=0.0024)$ and was larger than that of the methadone, fentanyl, and hydromorphone groups. Regarding safety, the discontinuation rate in the tapentadol group was the lowest of all groups (tapentadol vs. methadone vs. oxycodone vs. fentanyl vs. hydromorphone, $0.0 \%$ vs. $\mathbf{6 . 3 \%}$ vs. $\mathbf{5 . 0} \%$ vs. $\mathbf{3 . 8 \%}$ vs. $\mathbf{1 0 . 0} \%$, respectively). This study suggests that tapentadol could be efficacious for cancer patients with NP, and a preferred option in cases that require immediate dose adjustment or for those at high risk for adverse effects. However, the pain intensity was evaluated without pain assessment scales specific to NP. Thus, we think that it is desirable to validate our findings using assessment scales, such as the painDETECT questionnaire in future.

Key words cancer pain management; neuropathic pain; clinical pharmacology; palliative medicine; opioid

\section{INTRODUCTION}

Neuropathic pain is caused by degeneration, rupture, injury, or ischemia of the central, spinal, and peripheral nerves. Although the guidelines for pharmacological management of neuropathic pain recommend the administration of opioids for cancer-related neuropathic pain, ${ }^{1}$ no significant differences in analgesic effects for cancer pain, including neuropathic pain relief, have been reported between different opioids. ${ }^{2,3)}$ Therefore, the proper order of selection of oxycodone, fentanyl, and hydromorphone is unclear, causing clinical uncertainty. Moreover, they act only as $\mu$-opioid receptor agonists, and are thus often ineffective in neuropathic pain. In such cases, a serotonin-noradrenaline reuptake inhibitor or an $N$-methyl-Daspartate (NMDA) receptor antagonist is also administered. ${ }^{1,4)}$

Meanwhile, it has been reported that methadone, which has $\mu$-opioid receptor stimulating properties and NMDA receptor antagonism, and also inhibits the reuptake of serotonin and noradrenaline, is more effective than fentanyl in managing neuropathic pain in head and neck cancer patients. ${ }^{5)}$ Thus, multi-action opioids are expected to be effective in treating neuropathic pain in cancer patients. Tapentadol has $\mu$-opioid receptor stimulating and noradrenaline reuptake inhibitory actions and is pharmacologically expected to be effective in managing neuropathic pain in cancer patients. In fact, tapentadol is approved by the U.S. Food and Drug Administration for the management of diabetic peripheral neuropathy. Howev- er, the use of tapentadol as an analgesic in neoplastic disease has only started very recently $\left.{ }^{6}\right)$ and administering tapentadol remains challenging because it is only available as a relatively large tablet; thus, it is used only by patients who can take it orally. Tapentadol is rarely used for treating neuropathic pain in cancer patients. Therefore, in this study of cancer patients, we aimed to investigate the efficacy of tapentadol in managing cancer pain, including neuropathic pain and to identify a proper order of selection of opioid drugs for cancer pain, including neuropathic pain.

\section{MATERIALS AND METHODS}

Setting and Patients This was a retrospective, singlecenter, five-group comparison study. The data of 29, 32, 20, 26 , and 20 Japanese patients with cancer, hospitalized in the Ashiya Municipal Hospital, who were administered tapentadol, methadone, oxycodone, fentanyl, or hydromorphone, respectively, for neuropathic pain, were collected between January 1, 2013, and December 31, 2019. The data of 3, 7, 2, 6, and 5 patients who started using other analgesics within $14 \mathrm{~d}$ after the start of tapentadol, methadone, oxycodone, fentanyl, or hydromorphone, respectively, were excluded, since using other analgesics may influence the efficacy of opioids for cancer pain, including neuropathic pain.

Data Collection The following data were collected from the medical records: age, sex, body mass index (BMI), East- 
ern Cooperative Oncology Group Performance Status (ECOG PS), primary cancer site, metastases, type of pain (somatic, visceral, or neuropathic pain), prior opioid drugs used, the morphine-equivalent daily dose (MEDD), aspartate transaminase, alanine transaminase, $\gamma$-glutamyl transpeptidase, serum creatinine, estimated glomerular filtration rate, blood urea nitrogen, types and use of opioid rescue drugs, and concomitant medications (acetaminophen, nonsteroidal anti-inflammatory drugs (NSAIDs), corticosteroids, $\mathrm{Ca}^{2+}$ channel $\alpha 2 \delta$ ligands, serotonin-norepinephrine reuptake inhibitor, tricyclic antidepressant) at the start of each opioid drug. The laboratory test values at the start of each opioid drug $( \pm 7 \mathrm{~d})$ were considered. If multiple laboratory tests were conducted within the duration, the result obtained closest to the start of each opioid drug was analyzed. The use of opioid rescue drugs was assessed before and after administering each opioid drug (on days 3, 5, 7, and 14). MEDD conversion strategy suggested by $\mathrm{Na}$ tional Comprehensive Cancer Network was used to determine the dose of opioids other than methadone, ${ }^{7)}$ and the dose of methadone was determined in accordance with the Japanese packaging instructions for methadone. For example, when MEDD of prior opioid drugs ranged from 60 to $160 \mathrm{mg} / \mathrm{d}$, the dose of methadone was $15 \mathrm{mg} / \mathrm{d}$. When MEDD of prior opioid drugs ranged from 161 to $390 \mathrm{mg} / \mathrm{d}$, the dose of methadone was $30 \mathrm{mg} / \mathrm{d}$. When the MEDD of the previously administered opioid drug was $391 \mathrm{mg} / \mathrm{d}$ or more, the dose of methadone was $45 \mathrm{mg} / \mathrm{d}$. In some patients, the appropriate starting dose of each opioid drug was adjusted according to patients' symptoms.

Assessment of Types of Pain The type of pain (somatic, visceral, or neuropathic) was generally classified according to the diagnosis in the medical record recorded by physicians at the start of treatment with each opioid drug. Where there was no diagnosis, the type of pain was classified based on the McGill pain questionnaire (Japanese version). ${ }^{8}$ For example, pain described as "sharp" or "stabbing" was considered somatic pain. Pain due to bone metastases that worsened during movement, chest wall infiltration of lung cancer, tingling pain due to chest wall invasion of lung cancer, skin ulceration due to breast cancer and local recurrence of head and neck cancer, and lumbar back pain due to cancer infiltrating the retroperitoneum and pelvic floor were also judged to be somatic pain. "Pressing" or "heavy" pain was considered visceral pain. Upper abdominal pain and back pain due to pancreatic, abdominal, and retroperitoneal lymph node metastases, the pain of liver capsule distension due to liver tumor, and abdominal pain with unclear localization accompanied by abdominal distention due to cancerous peritonitis were judged to be visceral pain, and they were often seen in digestive system cancer. In addition, "allodynia" or "numbness" pain was considered neuropathic pain. Pain like an electric shock, and burning pain in the innervated area of the spinal cord due to metastasis and invasion of spinal bone and sensory disorders accompanied by numbness in the innervated area due to cancer or lymph node metastasis invading the cervical vertebrae, arm, or lumbosacral plexus, were judged to be neuropathic pain.

Assessment of Pain Intensity The pain scores on the numerical rating scale (NRS) or verbal rating scale (VRS) were investigated before and after the administration of each opioid drug (on days 3, 5, 7, and 14). The pain intensity was reported by the patients. NRS scores were converted to VRS scores as follows: NRS $0=$ no pain (VRS 0), NRS $1-4=$ mild pain (VRS 1), NRS 5-7= moderate pain (VRS 2), and NRS $8-10=$ severe pain $($ VRS 3$){ }^{9}{ }^{\text {) }}$

Primary and Secondary Endpoints The primary endpoint was the change in VRS scores before and $7 \mathrm{~d}$ after the administration of each opioid drug. The secondary endpoint was the discontinuation rate due to adverse effects.

Statistical Analysis One-way ANOVA was conducted for the comparisons of age and BMI. The chi-square test was used to compare the ratios of sex and types of pain among the five groups. The Kruskal-Wallis test was conducted for the comparisons of the ECOG PS, MEDD, and laboratory test values. The changes in VRS scores and the use of opioid rescue drugs were analyzed using the Steel-Dwass multiple comparison test. The required sample size was determined to detect an effect size of 0.80 in VRS scores between days 0 and 7 using a sample size calculator for a paired $t$-test. Assuming a statistical probability at a $5 \%$ level with a power of 0.80 , the minimum sample size was determined to be 15 . All statistical analyses were performed using BellCurve for Excel (Social Survey Research Information Co., Ltd., Tokyo, Japan). A twotailed $p$-value of $<0.05$ was considered statistically significant.

Ethical Approval This study was conducted in accordance with the ethical principles for medical research outlined in the Declaration of Helsinki, 1964, and per subsequent revisions and approved by the Ethical Review Boards of Ashiya Municipal Hospital (IRB Approval Code No. 25) and Osaka University Graduate School of Pharmaceutical Sciences (IRB Approval Code No. 30-10). Written consent to publish this study was obtained from all the patients.

\section{RESULTS}

The baseline characteristics of the patients are provided in Table 1. The mean \pm standard deviation MEDDs of the patients in each group were $30.8 \pm 42.0,114.2 \pm 104.6$, $19.2 \pm 33.2,21.9 \pm 28.7$, and $17.4 \pm 27.8 \mathrm{mg}$, respectively, and the MEDD was significantly higher in the methadone group than in the other four groups $(p<0.001)$. Regarding the use of concomitant medications at the start of the administration of each opioid drug, $16(55.2 \%), 17(53.1 \%), 10(50.0 \%), 15$ $(57.7 \%)$, and $7(35.0 \%)$ patients used NSAIDs, and $19(65.5 \%)$, $22(68.8 \%), 14(70.0 \%), 16(61.5 \%)$, and $11(55.0 \%)$ patients used adjuvant analgesics in the tapentadol, methadone, oxycodone, fentanyl, and hydromorphone groups, respectively. No significant differences were observed between the five groups in either liver or kidney function.

A total of $25(86.2 \%), 24(75.0 \%), 15(75.0 \%), 22(84.6 \%)$, and $16(80.0 \%)$ patients continued to use tapentadol, methadone, oxycodone, fentanyl, and hydromorphone, respectively, until day 7. The changes in the mean VRS scores before and after administration (on days 3, 5, 7, and 14) in each group are shown in Fig. 1. Before the administration of each opioid drug, the mean VRS scores (95\% confidence interval (CI)) in the tapentadol, methadone, oxycodone, fentanyl, and hydromorphone groups were 1.64 (1.37-1.91), 1.63 (1.39-1.87), 1.63 (1.32-1.94), 1.61 (1.34-1.88), and 1.59 (1.29-1.88), respectively. In the tapentadol group, the VRS score gradually decreased on day 3 (mean, 0.68; 95\% CI, 0.49-0.87), day 5 (mean, 0.65; 95\% CI, 0.45-0.85), day 7 (mean, 0.52; 95\% CI, 0.31-0.74), and day 14 (mean, 0.41; 95\% CI, 0.17-0.65). The VRS score 
Table 1. Baseline Patient Characteristics

\begin{tabular}{|c|c|c|c|c|c|c|}
\hline & $\begin{array}{l}\text { Tapentadol } \\
\quad(n=29)\end{array}$ & $\begin{array}{l}\text { Methadone } \\
\quad(n=32)\end{array}$ & $\begin{array}{l}\text { Oxycodone } \\
\quad(n=20)\end{array}$ & $\begin{array}{l}\text { Fentanyl } \\
(n=26)\end{array}$ & $\begin{array}{l}\text { Hydromorphone } \\
\qquad(n=20)\end{array}$ & $p$-Value \\
\hline Age (years), mean \pm S.D. (range) & $\begin{array}{l}69.9 \pm 13.8 \\
(49-92)\end{array}$ & $\begin{array}{l}69.3 \pm 12.4 \\
(40-87)\end{array}$ & $\begin{array}{l}75.4 \pm 6.2 \\
(64-89)\end{array}$ & $\begin{array}{l}72.9 \pm 13.0 \\
(48-93)\end{array}$ & $\begin{array}{l}75.8 \pm 13.1 \\
(43-93)\end{array}$ & $0.18^{a)}$ \\
\hline Sex, male, $n(\%)$ & $14(48.3)$ & $10(31.3)$ & $8(40.0)$ & $13(50.0)$ & $7(35.0)$ & $0.55^{b)}$ \\
\hline BMI $\left(\mathrm{kg} / \mathrm{m}^{2}\right)$, mean \pm S.D. (range) & $\begin{array}{c}19.9 \pm 4.3 \\
(12.3-29.7)\end{array}$ & $\begin{array}{c}18.8 \pm 2.5 \\
(14.2-23.7)\end{array}$ & $\begin{array}{c}19.5 \pm 3.1 \\
(13.6-24.4)\end{array}$ & $\begin{array}{c}18.9 \pm 3.2 \\
(14.4-25.4)\end{array}$ & $\begin{array}{c}20.9 \pm 4.9 \\
(14.2-33.1)\end{array}$ & $0.66^{a)}$ \\
\hline \multicolumn{7}{|l|}{ ECOG PS, $n(\%)$} \\
\hline 4 & $5(17.2)$ & $5(15.6)$ & $3(15.0)$ & $6(23.1)$ & $5(25.0)$ & $0.28^{c)}$ \\
\hline 3 & $10(34.5)$ & $19(59.4)$ & $5(25.0)$ & $9(34.6)$ & $6(30.0)$ & \\
\hline 2 & $6(20.7)$ & $5(15.6)$ & $3(15.0)$ & $4(15.4)$ & $2(10.0)$ & \\
\hline 1 & $4(13.8)$ & $2(6.3)$ & $5(25.0)$ & $4(15.4)$ & $3(15.0)$ & \\
\hline 0 & $4(13.8)$ & $1(3.1)$ & $4(20.0)$ & $3(11.5)$ & $4(20.0)$ & \\
\hline \multicolumn{7}{|l|}{ Primary cancer site, $n(\%)$} \\
\hline Colon & $6(20.7)$ & 7 (21.9) & $2(10.0)$ & $4(15.4)$ & $4(20.0)$ & N.A. \\
\hline Blood & $5(17.2)$ & $1(3.1)$ & $2(10.0)$ & $0(0.0)$ & $4(20.0)$ & \\
\hline Lung & $4(13.8)$ & $5(15.6)$ & $4(20.0)$ & $5(19.2)$ & $1(5.0)$ & \\
\hline Pancreas & $4(13.8)$ & $5(15.6)$ & $2(10.0)$ & $2(7.7)$ & $5(25.0)$ & \\
\hline Breast & $2(6.9)$ & $4(12.5)$ & $2(10.0)$ & $2(7.7)$ & $0(0.0)$ & \\
\hline Stomach & $2(6.9)$ & $0(0.0)$ & $2(10.0)$ & $4(15.4)$ & $0(0.0)$ & \\
\hline Uterine & $1(3.4)$ & $3(9.4)$ & $1(5.0)$ & $2(7.7)$ & $1(5.0)$ & \\
\hline Others & $5(17.2)$ & $7(21.9)$ & $5(25.0)$ & $7(26.9)$ & $5(25.0)$ & \\
\hline \multicolumn{7}{|l|}{$\begin{array}{l}\text { Metastases, } n(\%) \text { (including } \\
\quad \text { duplicate answers) }\end{array}$} \\
\hline Bone & $11(37.9)$ & $15(46.9)$ & $11(55.0)$ & $6(23.1)$ & $6(30.0)$ & N.A. \\
\hline Liver & $9(31.0)$ & $9(28.1)$ & $5(25.0)$ & $6(23.1)$ & $9(45.0)$ & \\
\hline Lung & $8(27.6)$ & $13(40.6)$ & $5(25.0)$ & $5(19.2)$ & $4(20.0)$ & \\
\hline Lymph node & $8(27.6)$ & $11(34.4)$ & $4(20.0)$ & $9(34.6)$ & $4(20.0)$ & \\
\hline Peritoneal & $6(20.7)$ & $4(12.5)$ & $2(10.0)$ & $5(19.2)$ & $3(15.0)$ & \\
\hline Brain & $3(10.3)$ & $4(12.5)$ & $1(5.0)$ & $3(11.5)$ & $1(5.0)$ & \\
\hline Small intestine & $2(6.9)$ & $0(0.0)$ & $0(0.0)$ & $0(0.0)$ & $0(0.0)$ & \\
\hline Pleura & $2(6.9)$ & $5(15.6)$ & $0(0.0)$ & $1(3.8)$ & $1(5.0)$ & \\
\hline Esophagus & $1(3.4)$ & $0(0.0)$ & $0(0.0)$ & $2(7.7)$ & $0(0.0)$ & \\
\hline Muscle & $0(0.0)$ & $3(9.4)$ & $0(0.0)$ & $0(0.0)$ & $1(5.0)$ & \\
\hline Pancreas & $0(0.0)$ & $2(6.3)$ & $1(5.0)$ & $0(0.0)$ & $0(0.0)$ & \\
\hline Subcutaneous tissue & $0(0.0)$ & $2(6.3)$ & $0(0.0)$ & $0(0.0)$ & $0(0.0)$ & \\
\hline Others & $5(17.2)$ & $8(25.0)$ & $1(5.0)$ & $5(19.2)$ & $4(20.0)$ & \\
\hline None & $2(6.9)$ & $1(3.1)$ & $0(0.0)$ & $2(7.7)$ & $0(0.0)$ & \\
\hline \multicolumn{7}{|l|}{$\begin{array}{l}\text { Type of pain, } n(\%) \text { (including } \\
\text { duplicate answers) }\end{array}$} \\
\hline Only neuropathic pain & $7(24.1)$ & $4(12.5)$ & $3(15.0)$ & $5(19.2)$ & $1(5.0)$ & $0.99^{b)}$ \\
\hline Somatic and neuropathic pain & $15(51.7)$ & $14(43.8)$ & $12(60.0)$ & $6(23.1)$ & $6(30.0)$ & \\
\hline Visceral and neuropathic pain & $3(10.3)$ & $3(9.4)$ & $3(15.0)$ & $6(23.1)$ & $8(40.0)$ & \\
\hline $\begin{array}{l}\text { Somatic, visceral, and neuro- } \\
\text { pathic pain }\end{array}$ & $4(13.8)$ & $11(34.4)$ & $2(10.0)$ & $9(34.6)$ & $5(25.0)$ & \\
\hline \multicolumn{7}{|l|}{$\begin{array}{l}\text { Opioids before switching to each } \\
\text { opioid, } n(\%) \text { (including dupli- } \\
\text { cate answers) }\end{array}$} \\
\hline Oxycodone & $9(31.0)$ & $8(25.0)$ & - & $9(34.6)$ & $3(15.0)$ & N.A. \\
\hline Fentanyl & $4(13.8)$ & $9(28.1)$ & $2(10.0)$ & - & $2(10.0)$ & \\
\hline Tapentadol & - & $5(15.6)$ & $2(10.0)$ & $1(3.8)$ & $2(10.0)$ & \\
\hline Morphine & $1(3.4)$ & $3(9.4)$ & $2(10.0)$ & $3(11.5)$ & $2(10.0)$ & \\
\hline Hydromorphone & $1(3.4)$ & $6(18.8)$ & $0(0.0)$ & $0(0.0)$ & - & \\
\hline Tramadol & $5(17.2)$ & $1(3.1)$ & $4(20.0)$ & $0(0.0)$ & $1(5.0)$ & \\
\hline Naïve & $9(31.0)$ & $1(3.1)$ & $10(50.0)$ & $13(50.0)$ & $10(50.0)$ & \\
\hline $\begin{array}{l}\text { MEDD }(\mathrm{mg} / \mathrm{d}), \text { mean } \pm \text { S.D. } \\
\quad \text { (range) }\end{array}$ & $\begin{array}{l}30.8 \pm 42.0 \\
(0-210)\end{array}$ & $\begin{array}{l}114.2 \pm 104.6 \\
\quad(0-500)\end{array}$ & $\begin{array}{l}19.2 \pm 33.2 \\
(0-120)\end{array}$ & $\begin{array}{l}21.9 \pm 28.7 \\
\quad(0-80)\end{array}$ & $\begin{array}{l}17.4 \pm 27.8 \\
\quad(0-105)\end{array}$ & $<0.001^{\mathrm{c}}$ \\
\hline \multicolumn{7}{|l|}{$\begin{array}{l}\text { Concomitant medications, } n(\%) \\
\quad \text { (including duplicate answers) }\end{array}$} \\
\hline Acetaminophen & $11(37.9)$ & $7(21.9)$ & $8(40.0)$ & $13(50.0)$ & $7(35.0)$ & N.A. \\
\hline \multicolumn{7}{|l|}{ NSAIDs } \\
\hline Loxoprofen & $9(31.0)$ & 7 (21.9) & $3(15.0)$ & 7 (26.9) & $4(20.0)$ & \\
\hline
\end{tabular}


Table 1. Continued

\begin{tabular}{|c|c|c|c|c|c|c|}
\hline & $\begin{array}{l}\text { Tapentadol } \\
(n=29)\end{array}$ & $\begin{array}{l}\text { Methadone } \\
(n=32)\end{array}$ & $\begin{array}{l}\text { Oxycodone } \\
\quad(n=20)\end{array}$ & $\begin{array}{l}\text { Fentanyl } \\
(n=26)\end{array}$ & $\begin{array}{l}\text { Hydromorphone } \\
\quad(n=20)\end{array}$ & $p$-Value \\
\hline Celecoxib & $5(17.2)$ & $8(25.0)$ & $3(15.0)$ & $5(19.2)$ & $2(10.0)$ & \\
\hline Diclofenac & $4(13.8)$ & $1(3.1)$ & $0(0.0)$ & $1(3.8)$ & $0(0.0)$ & \\
\hline Others & $1(3.4)$ & $3(9.4)$ & $2(10.0)$ & $5(19.2)$ & $1(5.0)$ & \\
\hline None & $13(44.8)$ & $15(46.9)$ & $10(50.0)$ & $11(42.3)$ & $13(65.0)$ & \\
\hline \multicolumn{7}{|l|}{ Adjuvant analgesics } \\
\hline Corticosteroids & $13(44.8)$ & $19(59.4)$ & $7(35.0)$ & $10(38.5)$ & $10(50.0)$ & \\
\hline $\mathrm{Ca}^{2+}$ channel $\alpha_{2} \delta$ ligands & $8(27.6)$ & $6(18.8)$ & $9(45.0)$ & $9(34.6)$ & $1(5.0)$ & \\
\hline SNRI & $1(3.4)$ & $5(15.6)$ & $1(5.0)$ & $2(7.7)$ & $0(0.0)$ & \\
\hline TCA & $0(0.0)$ & $1(3.1)$ & $0(0.0)$ & $0(0.0)$ & $0(0.0)$ & \\
\hline Others & $5(17.2)$ & $6(18.8)$ & $2(10.0)$ & $4(15.4)$ & $0(0.0)$ & \\
\hline None & $10(34.5)$ & $10(31.3)$ & $6(30.0)$ & $10(38.5)$ & $9(45.0)$ & \\
\hline \multicolumn{7}{|c|}{$\begin{array}{l}\text { Laboratory values before the ad- } \\
\text { ministration of opioids, median } \\
\text { (IQR) }\end{array}$} \\
\hline $\operatorname{AST}(\mathrm{U} / \mathrm{L})$ & $22.0(9.0-100.0)$ & $26.0(14.0-162.0)$ & $30.0(11.0-138.0)$ & $22.5(14.0-163.0)$ & $38.0(19.0-341.0)$ & $0.15^{c)}$ \\
\hline ALT (U/L) & $17.0(5.0-63.0)$ & $15.0(6.0-142.0)$ & $12.0(5.0-511.0)$ & $14.5(4.0-203.0)$ & $21.0(12.0-178.0)$ & $0.20^{c)}$ \\
\hline$\gamma$-GTP $(\mathrm{U} / \mathrm{L})$ & $59.0(16.0-562.0)$ & $104.0(17.0-457.0)$ & $35.0(15.0-327.0)$ & $31.5(12.0-2458.0)$ & $94.0(17.0-2377.0)$ & $0.22^{c)}$ \\
\hline $\operatorname{Scr}(\mathrm{mg} / \mathrm{dL})$ & $0.6(0.3-1.2)$ & $0.6(0.3-1.4)$ & $0.6(0.4-1.0)$ & $0.7(0.2-1.4)$ & $0.7(0.4-1.2)$ & $0.22^{c)}$ \\
\hline eGFR (mL/min) & $94.5(47.2-169.4)$ & $86.4(28.0-156.2)$ & $81.6(44.0-148.1)$ & $75.3(36.3-277.6)$ & $64.9(46.7-136.5)$ & $0.23^{c)}$ \\
\hline BUN (mg/dL) & $16.8(5.4-84.3)$ & $14.1(5.4-50.0)$ & $13.4(6.4-26.6)$ & $20.9(5.9-37.5)$ & $14.9(6.5-35.6)$ & $0.26^{c)}$ \\
\hline
\end{tabular}

a) Single-factor ANOVA; b) chi-square for independence test; c) Kruskal-Wallis test. Abbreviations: S.D., standard deviation; BMI, body mass index; ECOG PS, Eastern Cooperative Oncology Group Performance Status; N.A., not available; MEDD, morphine-equivalent daily dose; NSAIDs, nonsteroidal anti-inflammatory drugs; SNRI, serotonin-norepinephrine reuptake inhibitor; TCA, tricyclic antidepressant; IQR, interquartile range; AST, aspartate transaminase; ALT, alanine transaminase; $\gamma$-GTP, $\gamma$-glutamyl transpeptidase; Scr, serum creatinine; eGFR, estimated glomerular filtration rate; BUN, blood urea nitrogen.
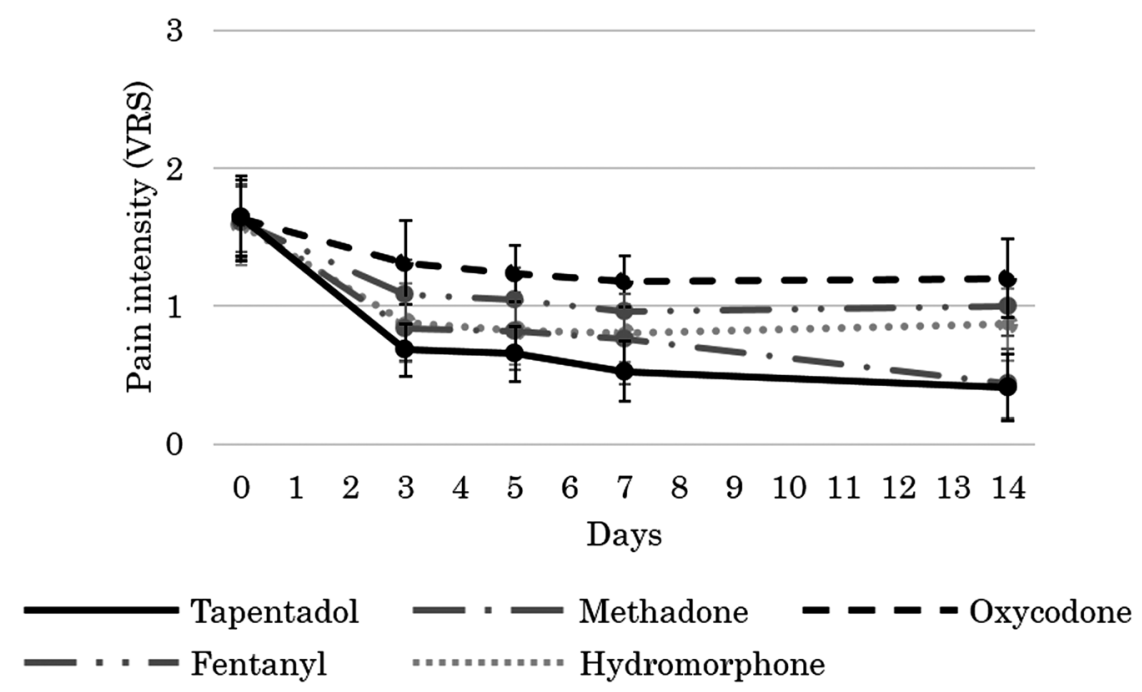

Fig. 1. Changes in the Mean Verbal Rating Scale Scores on Pain

The verbal rating scale (VRS) scores were determined before tapentadol, methadone, oxycodone, fentanyl, or hydromorphone administration, and on days 3, 5, 7, and 14 after tapentadol, methadone, oxycodone, fentanyl, or hydromorphone administration. NRS scores were converted to VRS scores as follows: NRS $0=$ no pain $($ VRS 0 ), NRS $1-4=$ mild pain (VRS 1$)$, NRS $5-7=$ moderate pain (VRS 2), and NRS $8-10=$ severe pain (VRS 3$)^{9}{ }^{9}$

in the methadone group also gradually decreased on day 3 (mean, 0.83; 95\% CI, 0.61-1.06), day 5 (mean, 0.82; 95\% CI, 0.54-1.10), day 7 (mean, 0.76; 95\% CI, 0.43-1.09), and day 14 (mean, $0.44 ; 95 \% \mathrm{CI}, 0.19-0.69$ ). In the oxycodone group, the VRS score decreased on day 3 (mean, 1.32; 95\% CI, 1.01-1.62), day 5 (mean, 1.24; 95\% CI, 1.03-1.44), and day 7 (mean, 1.18; 95\% CI, 0.99-1.36), and was maintained until day 14 (mean, $1.20 ; 95 \%$ CI, 0.92-1.48). In the fentanyl group, the VRS score decreased on day 3 (mean, 1.09; 95\% CI, 0.84-1.33), day 5 (mean, 1.04; 95\% CI, 0.81-1.27), and day 7 (mean, 0.96; 95\% CI, 0.76-1.15), and there was almost no change in the VRS score between day 7 and day
14 (mean, 1.00; 95\% CI, 0.79-1.21). In the hydromorphone group, the VRS score decreased on day 3 (mean, $0.88 ; 95 \%$ CI, 0.60-1.17), day 5 (mean, 0.82;95\% CI, 0.57-1.07), and day 7 (mean, $0.80 ; 95 \%$ CI, 0.59-1.01), and remained nearly unchanged on day 14 (mean, 0.87 ; 95\% CI, 0.61-1.13).

There was no significant difference in the VRS scores before administering each opioid drug among all groups $(p=0.99)$. Table 2 shows the differences in the VRS scores on days $3,5,7$, and 14 before and after administration.

The reductions in the VRS scores were significantly larger with the use of tapentadol on days 3, 5, 7, and 14 than with oxycodone (day 3: $p=0.017$; day 5: $p=0.012$; day 7: 
$p=0.0024$; day $14: p=0.035$ ). Although no statistically significant differences were observed between the tapentadol and fentanyl groups in terms of the reductions in the VRS scores on days $3,5,7$, and 14 , the reductions in the VRS scores were larger with the use of tapentadol than with fentanyl (day 3: $p=0.19$; day 5: $p=0.22$; day 7: $p=0.093$; day 14: $p=0.13$ ). Comparison of the reductions in the VRS scores between the tapentadol and hydromorphone groups revealed no statistically significant differences on days $3,5,7$, and 14 , but the reductions in the VRS scores were greater in the tapentadol group than in the hydromorphone group (day 3: $p=0.82$; day 5: $p=0.86$; day 7: $p=0.15$; day 14: $p=0.31$ ). Comparing the VRS score reductions between the methadone and oxycodone groups revealed no significant differences on days 3,5 , and 7 , but the reduction in the VRS score on day 14 was significantly larger in the methadone group than in the oxycodone group (day 3: $p=0.063$; day 5: $p=0.20$; day $7: p=0.31$; day 14: $p=0.029)$. No significant differences were observed between the tapentadol and methadone groups in terms of the reductions in the VRS scores on days 3, 5, 7, and 14 (day 3: $p=0.96$; day 5: $p=0.96$; day 7: $p=0.91$; day 14: $p=0.99$ ).

The change in the use of opioid rescue drugs is presented in
Fig. 2. There was no significant difference in the average use of opioid rescue drugs at the start of the administration (day 0 ) of each opioid drug among the tapentadol (mean, 2.37; 95\% CI, 1.25-3.48), methadone (mean, 3.32; 95\% CI, 2.57-4.07), oxycodone (mean, 1.09; 95\% CI, 0.53-1.65), fentanyl (mean, 2.50; 95\% CI, 1.33-3.67), and hydromorphone (mean, 1.45; $95 \%$ CI, $0.53-2.38)$ groups ( $p=0.99$ ).

The reductions in the average use of opioid rescue drugs on days $3,5,7$, and 14 compared to those before the administration of each opioid drug are provided in Table 3. Although the reductions in the average use on days $3,5,7$, and 14 were greater in the tapentadol group than in the oxycodone group, no significant differences were observed between the two groups (day 3: $p=0.31$; day 5: $p=0.46$; day $7: p=0.34$; day 14: $p=0.31$ ). Comparison of the reductions in the average use of opioid rescue drugs between the tapentadol and fentanyl groups revealed no significant differences on days 3, 5, 7, and 14 (day 3: $p=0.68$; day 5: $p=0.95$; day 7: $p=0.96$; day 14: $p=0.96$ ). There were no significant differences in the reductions in the average use of opioid rescue drugs between the tapentadol and hydromorphone groups on days 3, 5, 7, and 14 (day 3: $p=0.76$; day 5: $p=0.91$; day 7: $p=0.96$;

Table 2. Differences in the Verbal Rating Scale Scores Compared to before Tapentadol, Methadone, Oxycodone, Fentanyl, or Hydromorphone Administration

\begin{tabular}{lcccc}
\hline \hline & Day 3 & Day 5 & Day 7 & Day 14 \\
\hline Tapentadol, mean $(95 \% \mathrm{CI})$ & $0.96(0.67-1.25)$ & $0.96(0.70-1.22)$ & $1.10(0.86-1.33)$ & $1.12(0.75-1.49)$ \\
Methadone, mean $(95 \% \mathrm{CI})$ & $0.83(0.55-1.11)$ & $0.86(0.49-1.24)$ & $0.90(0.48-1.33)$ & $1.13(0.73-1.52)$ \\
Oxycodone, mean $(95 \% \mathrm{CI})$ & $0.32(0.10-0.53)$ & $0.29(0.07-0.52)$ & $0.35(0.12-0.59)$ & $0.33(0.09-0.58)$ \\
Fentanyl, mean $(95 \% \mathrm{CI})$ & $0.52(0.31-0.73)$ & $0.57(0.36-0.77)$ & $0.65(0.42-0.89)$ & $0.53(0.30-0.76)$ \\
Hydromorphone, mean $(95 \% \mathrm{CI})$ & $0.71(0.48-0.93)$ & $0.76(0.56-0.97)$ & $0.67(0.42-0.91)$ & $0.60(0.28-0.92)$ \\
\hline$p$-Value (tapentadol $v s$. oxycodone) & 0.017 & 0.012 & 0.0024 & 0.093 \\
$p$-Value (tapentadol $v s$. fentanyl) & 0.19 & 0.22 & 0.15 & 0.13 \\
$p$-Value (tapentadol $v$ s. hydromorphone) & 0.82 & 0.86 & 0.91 & 0.31 \\
$p$-Value (tapentadol $v s$. methadone) & 0.96 & 0.96 & 0.31 & 0.99 \\
$p$-Value (methadone $v s$. oxycodone) & 0.063 & 0.20 & 0.029 \\
\hline
\end{tabular}

Abbreviations: CI, confidence interval; vs., versus.
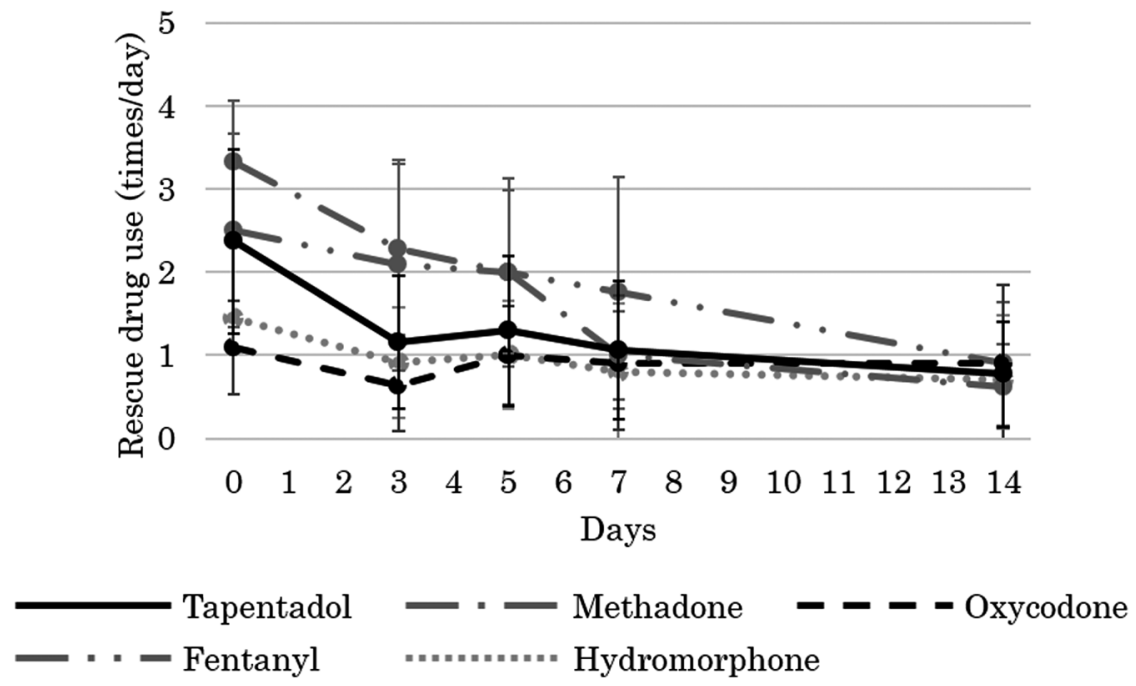

Fig. 2. Changes in the Mean Use of Opioid Rescue Drugs

The use of opioid rescue drugs was investigated before tapentadol, methadone, oxycodone, fentanyl, or hydromorphone administration, and on days 3,5 , 7 , and 14 after starting tapentadol, methadone, oxycodone, fentanyl, or hydromorphone. When multiple types of opioid rescue drugs were used, the total number of times the opioid rescue drugs were used was recorded. 
Table 3. Changes in the Use of Opioid Rescue Drugs as Compared to before Tapentadol, Methadone, Oxycodone, Fentanyl, or Hydromorphone Administration

\begin{tabular}{|c|c|c|c|c|}
\hline & Day 3 & Day 5 & Day 7 & Day 14 \\
\hline Tapentadol, mean $(95 \% \mathrm{CI})$ & $1.21(0.51-1.91)$ & $1.00(0.13-1.87)$ & $1.31(-0.10-2.73)$ & $1.92(0.38-3.47)$ \\
\hline Methadone, mean $(95 \% \mathrm{CI})$ & $1.32(0.68-2.00)$ & $1.60(0.87-2.33)$ & $2.60(1.79-3.41)$ & $3.00(2.12-3.88)$ \\
\hline Oxycodone, mean (95\% CI) & $0.46(-0.16-1.07)$ & $0.091(-0.40-0.58)$ & $0.18(-0.33-0.70)$ & $0.20(-0.71-1.11)$ \\
\hline Fentanyl, mean $(95 \% \mathrm{CI})$ & $0.42(-0.62-1.45)$ & $0.50(-0.32-1.32)$ & $0.75(-0.48-1.98)$ & $1.20(0.29-2.11)$ \\
\hline Hydromorphone, mean $(95 \% \mathrm{CI})$ & $0.55(-0.07-1.16)$ & $0.45(-0.40-1.31)$ & $0.60(-0.95-2.15)$ & $0.70(0.04-1.36)$ \\
\hline$p$-Value (tapentadol vs. oxycodone) & 0.31 & 0.46 & 0.34 & 0.31 \\
\hline$p$-Value (tapentadol vs. fentanyl) & 0.68 & 0.95 & 0.96 & 0.96 \\
\hline$p$-Value (tapentadol vs. hydromorphone) & 0.76 & 0.91 & 0.96 & 0.58 \\
\hline$p$-Value (tapentadol vs. methadone) & 0.99 & 0.91 & 0.42 & 0.55 \\
\hline$p$-Value (methadone $v s$. oxycodone) & 0.14 & 0.074 & 0.0045 & 0.0093 \\
\hline
\end{tabular}

Abbreviations: CI, confidence interval; vs., versus.

Table 4. Reasons for Discontinuing Methadone, Oxycodone, Fentanyl, or Hydromorphone

\begin{tabular}{lclcr}
\hline \hline Drugs & $\begin{array}{c}\text { Patient } \\
\text { No. }\end{array}$ & $\begin{array}{c}\text { Reasons for } \\
\text { discontinuation }\end{array}$ & $\begin{array}{c}\text { Duration since } \\
\text { the start of drug } \\
\text { administration }\end{array}$ & Dose \\
\hline Methadone & 1 & Nausea & $2 \mathrm{~d}$ & $15 \mathrm{mg} / \mathrm{d}$ \\
Oxycodone & 2 & Over-sedation & $3 \mathrm{~d}$ & $15 \mathrm{mg} / \mathrm{d}$ \\
Fentanyl & 1 & Constipation & $3 \mathrm{~d}$ & $20 \mathrm{mg} / \mathrm{d}$ \\
$\begin{array}{c}\text { Hydromor- } \\
\text { phone }\end{array}$ & 1 & Over-sedation & $7 \mathrm{~d}$ & $2 \mathrm{mg} / \mathrm{d}$ \\
\multicolumn{1}{c}{ Nonsea } & $5 \mathrm{~d}$ & $4 \mathrm{mg} / \mathrm{d}$ \\
\hline
\end{tabular}

day 14: $p=0.58$ ). Comparing the reductions in the average use of opioid rescue drugs between the methadone and oxycodone groups revealed no significant differences on days 3 and 5 , but the reductions on days 7 and 14 were significantly greater in the methadone group than in the oxycodone group (day 3: $p=0.14$; day 5: $p=0.074$; day 7: $p=0.0045$; day 14 : $p=0.0093)$. Furthermore, there was no change in the use of NSAIDs and adjuvant analgesics for the relief of pain in all groups.

Six patients discontinued the opioid drugs within $7 \mathrm{~d}$ (Table 4). In the tapentadol group, no patients discontinued the drugs. Two patients $(6.3 \%)$ discontinued methadone due to nausea $(n=1)$ and over-sedation $(n=1)$, one patient $(5.0 \%)$ discontinued oxycodone due to constipation, one patient (3.8\%) discontinued fentanyl due to over-sedation, and two patients $(10.0 \%)$ discontinued hydromorphone due to nausea $(n=1)$ and over-sedation $(n=1)$.

\section{DISCUSSION}

To the best of our knowledge, this is the first study to compare the efficacy of tapentadol to that of other opioids in treating cancer pain, including neuropathic pain. In this study, both VRS scores and the average use of opioid rescue drugs in the methadone group on day 7 were significantly lower than those on day 0 . This result was consistent with previous studies and suggested that methadone may be effective in cancer patients with neuropathic pain. ${ }^{5)}$ Moreover, the VRS score reduction on day 7 in the tapentadol group was similar to that of the methadone group and larger than that of the oxycodone, fentanyl, and hydromorphone groups. These results suggest that tapentadol may be as effective as methadone and superior to oxycodone, fentanyl, and hydromorphone in managing cancer pain, including neuropathic pain, within $14 \mathrm{~d}$ after the start of each opioid drug.

The reductions in the VRS scores and average uses of opioid rescue drugs in the tapentadol group were greater than those of the oxycodone, fentanyl, and hydromorphone groups. This may be related to the noradrenaline reuptake inhibition by tapentadol. It has been demonstrated in neuropathic pain that the functional downregulation or desensitization of $\mu$-opioid receptors in the dorsal horn of the spinal cord occurs, and the analgesic effect of opioids is diminished. ${ }^{10)}$ In addition, it has been reported that the sprouting of noradrenergic nerve fibers in the spinal cord increases ${ }^{11,12)}$; presynaptic and postsynaptic $\alpha_{2}$-adrenoceptor activity in the spinal dorsal horn increases, and the function of the descending noradrenergic inhibitory system is impaired in the neuropathic pain state. ${ }^{13)}$ Furthermore, it has been suggested that the analgesic effect of tapentadol is more sensitive to noradrenaline reuptake inhibition than $\mu$-opioid receptor stimulation in nerve-injured rats. ${ }^{14)}$ Therefore, the dual actions of tapentadol, including $\mu$-opioid receptor stimulation and noradrenaline reuptake inhibition, exert an analgesic effect on cancer pain, including neuropathic pain, superior to oxycodone, fentanyl, and hydromorphone, which have only the $\mu$-opioid receptor stimulating action. Although there is evidence for the efficacy of oxycodone, fentanyl, and hydromorphone, ${ }^{15-17)}$ most studies used a placebo for comparison. Thus, there is insufficient evidence on the selection guidelines for opioids for neuropathic pain, and this study is significant in its comparison of the effects of multiple opioids on neuropathic pain.

The VRS score reduction in the tapentadol group was similar to that of the methadone group, which is effective for neuropathic pain, on day 14 and was greater on days 3, 5, and 7. This could be affected by the regulations regarding dosing changes in methadone in Japan. Japanese packaging instructions for methadone prohibit a dose-escalation within $7 \mathrm{~d}$ of the start of the medication, as this may result in overdose; thus, there was little change in the VRS score within $7 \mathrm{~d}$ in the methadone group in this study. However, tapentadol can be titrated in increments of $50 \mathrm{mg}$ every $3 \mathrm{~d}$ to an effective dose. ${ }^{18)}$ The tapentadol dose was adjusted more than once within $7 \mathrm{~d}$ in 10 of 29 patients $(34.5 \%)$ in the tapentadol group. As high opioid doses are often needed for neuropathic pain, it is desirable to increase the dose rapidly. ${ }^{19)}$ If further studies suggest 
that tapentadol is effective in treating cancer pain, including neuropathic pain, tapentadol will be an attractive alternative to methadone. Unlike methadone, a suitable dose of tapentadol can easily be achieved soon after initiation. Comparable pharmacokinetics of tapentadol were shown between Japanese and Western patients. ${ }^{20}$ On the other hand, methadone is mainly metabolized by CYP3A4 and 2B6, and the influence of CYP2B6 genotype is important in considering ethnic differences. ${ }^{21)} \mathrm{CYP} 2 \mathrm{~B} 6 * 6$ is reported to reduce the expression and activity of CYP2B6 significantly ${ }^{22)}$; the incidence of CYP2B6*6 tends to be lower in Japanese than in Western patients. ${ }^{23)}$ Taking these reports into consideration, it is expected that the clearance of methadone in Japanese individuals will increase. However, in this study, the reduction in the VRS score and the average use of opioid rescue drugs on day 14 was significantly larger in the methadone group than in the oxycodone group. This result suggests that the analgesic effect of methadone was sufficiently obtained.

When the number of times the opioid rescue drugs are used is significantly higher in one group than in other groups, it may be impossible to accurately evaluate the effect of the regular opioid; therefore, we investigated the number of times the opioid rescue drugs were used. The use of opioid rescue drugs decreased to less than 1 time/d within $14 \mathrm{~d}$ after the start of each opioid drug in all groups. This result suggests that the differences in VRS score reduction among the five groups might be due to the analgesic effects of the opioids rather than the analgesic effects of the opioid rescue drugs.

Regarding tolerability, no patient discontinued tapentadol due to adverse effects; among the five groups, the rate of discontinuation due to adverse effects was the lowest in the tapentadol group. Tapentadol is known to be well tolerated in terms of gastrointestinal adverse events because it has a reduced affinity for the $\mu$-opioid receptor. ${ }^{24)}$ Furthermore, tapentadol has a more favorable adverse effect profile than other opioids because it has low plasma protein binding activity and is not metabolized by the CYP system. ${ }^{25)}$ Therefore, it is suggested that tapentadol is a viable treatment option even in patients who are at high risk of adverse effects or drug interactions.

There are several limitations to this study. First, the pain intensity was evaluated without the use of pain assessment scales specific to neuropathic pain, and other types of pain (such as somatic or visceral pain) might have affected the outcomes. Thus, it is desirable to validate our findings using assessment scales, such as the painDETECT questionnaire, for evaluating neuropathic pain. Second, although there were no significant differences in the baseline patient characteristics (shown in Table 1), confounding for patients' backgrounds among the five groups should not be eliminated since this was a retrospective study. Third, this study was carried out among only Japanese and the generalizability of these results to other population, particularly Westerners may be limited. Therefore, to validate our findings, we recommend conducting further large-scale studies.

In conclusion, this study suggests that tapentadol is as effective as methadone and is superior to oxycodone, fentanyl, and hydromorphone in treating cancer pain, including neuropathic pain, within $14 \mathrm{~d}$ after the start of each opioid drug. Moreover, tapentadol would be a preferred option for neuropathic pain in cancer patients in need of an immediate dose increase or who have a high risk of adverse effects.

Conflict of Interest The authors declare no conflict of interest.

\section{REFERENCES}

1) Japanese Society of Pain Clinicians. Guideline for the pharmacologic management of neuropathic pain. Shinko Trading Press, Tokyo (2016).

2) Wiffen PJ, Wee B, Derry S, Bell RF, Moore RA. Opioids for cancer pain-an overview of Cochrane reviews. Cochrane Database Syst. Rev., 7, CD012592 (2017).

3) Nosek K, Leppert W, Nosek H, Wordliczek J, Onichimowski D. A comparison of oral controlled-release morphine and oxycodone with transdermal formulations of buprenorphine and fentanyl in the treatment of severe pain in cancer patients. Drug Des. Dev. Ther. 11, 2409-2419 (2017)

4) Fallon MT. Neuropathic pain in cancer. Br. J. Anaesth., 111, 105111 (2013).

5) Haumann J, Geurts JW, van Kuijk SM, Kremer B, Joosten EA, van den Beuken-van Everdingen MH. Methadone is superior to fentanyl in treating neuropathic pain in patients with head-and-neck cancer. Eur. J. Cancer, 65, 121-129 (2016).

6) Carmona-Bayonas A, Jiménez Fonseca P, Virizuela Echaburu J. Tapentadol for cancer pain management: a narrative review. Pain Pract., 17, 1075-1088 (2017).

7) Swarm RA, Paice JA, Anghelescu DL, et al. Adult cancer pain, version 3.2019, NCCN clinical practice guidelines in oncology. J. Natl. Compr. Canc. Netw., 17, 977-1007 (2019).

8) Satow A, Nakatani K, Taniguchi S. Analysis of perceptual characteristics of pain describing in words caused by occupational cervicobrachial disorder and similar disease. Jpn. Psychol. Res., 30, 132-143 (1988).

9) Paul SM, Zelman DC, Smith M, Miaskowski C. Categorizing the severity of cancer pain: further exploration of the establishment of cutpoints. Pain, 113, 37-44 (2005).

10) Smith HS. Opioids and neuropathic pain. Pain Physician, 15 (3 Suppl.), ES93-ES110 (2012).

11) Ma W, Eisenach JC. Chronic constriction injury of sciatic nerve induces the up-regulation of descending inhibitory noradrenergic innervation to the lumbar dorsal horn of mice. Brain Res., 970, $110-118$ (2003).

12) Hayashida KI, Clayton BA, Johnson JE, Eisenach JC. Brain derived nerve growth factor induces spinal noradrenergic fiber sprouting and enhances clonidine analgesia following nerve injury in rats. Pain, 136, 348-355 (2008).

13) Chen SR, Chen H, Yuan WX, Pan HL. Increased presynaptic and postsynaptic $\alpha_{2}$-adrenoceptor activity in the spinal dorsal horn in painful diabetic neuropathy. J. Pharmacol. Exp. Ther., 337, 285-292 (2011).

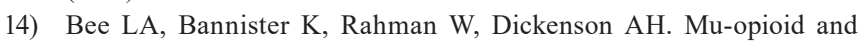
noradrenergic $\alpha(2)$-adrenoceptor contributions to the effects of tapentadol on spinal electrophysiological measures of nociception in nerve-injured rats. Pain, 152, 131-139 (2011).

15) Gaskell H, Derry S, Stannard C, Moore RA. Oxycodone for neuropathic pain in adults. Cochrane Database Syst. Rev., 7, CD010692 (2016).

16) Derry S, Stannard C, Cole P, Wiffen PJ, Knaggs R, Aldington D, Moore RA. Fentanyl for neuropathic pain in adults. Cochrane Database Syst. Rev., 10, CD011605 (2016).

17) Stannard C, Gaskell H, Derry S, Aldington D, Cole P, Cooper TE, Knaggs R, Wiffen PJ, Moore RA. Hydromorphone for neuropathic pain in adults. Cochrane Database Syst. Rev., 5, CD011604 (2016).

18) Sánchez Del Águila MJ, Schenk M, Kern KU, Drost T, Steigerwald I. Practical considerations for the use of tapentadol prolonged re- 
lease for the management of severe chronic pain. Clin. Ther., 37, 94-113 (2015).

19) Pergolizzi J, Böger RH, Budd K, Dahan A, Erdine S, Hans G, Kress HG, Langford R, Likar R, Raffa RB, Sacerdote P. Opioids and the management of chronic severe pain in the elderly: consensus statement of an International Expert Panel with focus on the six clinically most often used World Health Organization Step III opioids (buprenorphine, fentanyl, hydromorphone, methadone, morphine, oxycodone). Pain Pract., 8, 287-313 (2008).

20) Zhang L, Yan X, Nobe S, Zannikos P, Etropolski M, Nandy P. Quantifying the exposure of tapentadol extended release in Japanese patients with cancer pain and bridging tapentadol pharmacokinetics across populations using a modeling approach. Clin. Drug Investig., 37, 273-283 (2017).

21) Kharasch ED, Hoffer C, Whittington D, Sheffels P. Role of hepatic and intestinal cytochrome P450 3A and 2B6 in the metabolism, disposition, and miotic effects of methadone. Clin. Pharmacol. Ther.,
76, 250-269 (2004).

22) Turpeinen M, Zanger UM. Cytochrome P450 2B6: function, genetics, and clinical relevance. Drug Metabol. Drug Interact., 27, 185-197 (2012).

23) Hiratsuka M, Takekuma Y, Endo N, Narahara K, Hamdy SI, Kishikawa Y, Matsuura M, Agatsuma Y, Inoue T, Mizugaki M. Allele and genotype frequencies of CYP2B6 and CYP3A5 in the Japanese population. Eur. J. Clin. Pharmacol., 58, 417-421 (2002).

24) Kress HG, Koch ED, Kosturski H, Steup A, Karcher K, Lange B, Dogan C, Etropolski MS, Eerdekens M. Tapentadol prolonged release for managing moderate to severe, chronic malignant tumorrelated pain. Pain Physician, 17, 329-343 (2014).

25) Vadivelu N, Huang Y, Mirante B, Jacoby M, Braveman FR, Hines RL, Sinatra R. Patient considerations in the use of tapentadol for moderate to severe pain. Drug Healthc. Patient Saf., 5, 151-159 (2013). 